\title{
Management of cancer-associated venous thrombosis
}

\author{
Ozlem Er' \\ Leo Zacharski \\ 'Department of Medical Oncology, \\ Erciyes University Medical Faculty, \\ Kayseri, Turkey; ${ }^{2}$ Department of \\ Medicine, Dartmouth Medical School, \\ Lebanon, New Hampshire, USA
}

\begin{abstract}
The association between malignancy and venous thromboembolic disease has been recognized for over a century and a half. During this time, a substantial body of literature has developed showing that malignancy is not only a hypercoagulable state characterized by an increased risk of thrombosis but also that components of blood coagulation reactions are capable of supporting tumor growth and dissemination. In recent years a succession of meticulously performed clinical trials has clarified optimal therapy intended to both prevent and treat thromboembolism that occurs in the setting of cancer. However, much remains to be accomplished in terms of practitioner education on the merits of optimal therapy. Of perhaps greater interest is the possibility that drugs capable of controlling cancer-associated hypercoagulability may provide a means for improving cancer survival while avoiding the toxicities characteristic of conventional anti-tumor therapy. Clearly, ample incentive exists for collaboration between basic and clinical scientists interested in improving the management of malignancy and its thromboembolic complications.
\end{abstract}

Keywords: cancer, dalteparin, low molecular weight heparin, venous thrombosis

\section{Introduction}

Venous thromboembolism (VTE) is a major problem in cancer patients that significantly and negatively affects both morbidity and mortality (Heit et al 1999; Lip et al 2002; Khorana 2003). Cancer and VTE have a two-way clinical correlation. For example, the presence of malignant or pre-malignant cells may trigger systemic coagulation activation that leads to VTE while an activated coagulation mechanism may impact malignant progression. Thromboembolism may be recognized at the time of first cancer diagnosis, may complicate the course of established malignancy or may occur well before the first recognition of cancer (Hettiarachchi et al 1998; Zacharski 2003; Monreal et al 2004). In this review, we will discuss the occurrence and clinical significance of cancer-associated VTE, the prophylaxis and treatment of VTE in cancer patients, and the potential anticancer effects of low molecular weight heparins.

\section{Thromboembolism in cancer patients Prevalence and clinical significance of venous thromboembolism in cancer patients}

VTE is common in cancer patients. Of all patients presenting with acute VTE, 15\% to $20 \%$ are associated clinically with malignancy; $2 \%-5 \%$ of these are diagnosed concurrently with cancer, and 5\%-10\% are diagnosed with cancer during follow-up. Clearly, cancer patients are at a higher risk of VTE than are patients without cancer. Levitan and colleagues (1999) examined the association between the diagnosis of cancer and the occurrence of VTE and showed that VTE was significantly more common in patients with versus without malignancy at the time of first hospitalization. Furthermore, the risk of readmission with recurrent VTE as well as death within 6 
months was significantly greater in patients with malignancy-associated VTE compared with VTE without malignancy. Some data are available regarding the incidence of VTE among patients with specific types of cancer (Chew et al 2006). The Medicare database showed highest cumulative incidence of VTE in patients with cancer of the ovary, brain, pancreas, and lymphoma followed by cancer of the gastrointestinal tract, leukemia, and lung (Levitan et al 1999). Analysis of large databases has indicated that cancer patients who either develop a VTE during the course of established cancer or are diagnosed with a thrombotic episode when cancer is first diagnosed have poorer survival compared with cancer patients without thrombosis (Sorensen et al 2000; Alcalay et al 2006)

Active cancer is associated with a hypercoagulable state that is attributable to the interaction of multiple mechanisms. Examples of such interacting mechanisms include venous stasis due to immobilization that commonly accompanies the poor performance status characteristic of malignancy, recent cancer surgery, release of procoagulant factors produced by the tumor cells or activated host macrophages, endothelial damage caused by central vein catheters, radiotherapy, chemotherapy or hormonal therapy. The complexity of this process is illustrated by the fact that alteration in several of these contributing factors together with activation of components of hemostatic pathways is likely to contribute to thrombosis in individual cancer patients (Lee and Levine 2002).

The strength and clinical importance of the association between cancer and thrombosis has led to several trials exploring the benefits of pharmacologic thromboprophylaxis in cancer patients.

\section{Thromboprophylaxis in cancer patients \\ Perioperative and prolonged thromboprophylaxis in cancer patients undergoing surgery}

The risk of postoperative VTE is approximately twice as high in cancer patients as in patients undergoing comparable surgery for benign conditions (Clagett and Reisch 1988). The increased risk of VTE in cancer patients undergoing surgery is comparable with that in other high-risk surgical procedures such as orthopedic surgery. Investigation into the optimal dose of low molecular weight heparin (LMWH) for prophylaxis during cancer surgery has shown that a "high prophylactic" dose of dalteparin, $5000 \mathrm{IU}$, administered once daily for 7 days is significantly more effective than $2500 \mathrm{IU}$, and is equally safe (Bergqvist et al 1995). Mismetti and colleagues (2001) have conducted a meta-analysis of trials that compared LMWH with unfractionated heparin (UFH) in high risk surgery and the results of these trials provide evidence that once daily LMWH is as safe and effective as multiple injections of UFH per day for the prophylaxis of postoperative DVT in patients with cancer.

In patients undergoing abdominal surgery, increased levels of D-dimer have been shown to persist for at least 14 days, with levels being significantly higher in cancer patients compared with patients without cancer (Galster et al 2000). The extended activation of the coagulation system in cancer suggests that patients undergoing major abdominal surgery are a particularly high-risk population who may benefit from prolonged thromboprophylaxis. Given the concept that the risk of VTE extends beyond the immediate postoperative period in patients undergoing cancer surgery, the Duration of Prophylaxis against Venous Thromboembolism with Enoxaparin After Surgery for Cancer (ENOXACAN II) investigators studied extended prophylaxis in cancer surgery (Bergqvist et al 2002). In this randomized, double-blind trial, patients undergoing surgery for abdominal malignancy received one week of enoxaparin and then were randomly assigned to enoxaparin or placebo for 21 more days. The incidence of late occurring VTE assessed by bilateral venography was $12 \%$ with placebo compared with $4.8 \%$ in the prolonged thromboprophylaxis group, a $60 \%$ relative risk reduction. These results are supported by data from a trial conducted by Rasmussen and colleagues (2003) This prospective, randomized multicenter clinical trial, FAME (prolonged thromboprophylaxis with Fragmin After Major Surgery), was designed to evaluate the efficacy and safety of 1 week compared with 4 weeks of thromboprophylaxis with dalteparin following major abdominal surgery. A subgroup analysis of 198 cancer patients entered in this study showed that extending thromboprophylaxis from 1 week to 4 weeks reduced significantly the incidence of venographically proven DVT from $19.6 \%$ to $8.8 \%$ and also reduced the incidence of proximal DVT. In both studies, the risk of bleeding was not increased with prolonged prophylaxis. These two studies support the use of extended thromboprophylaxis with LMWH after major surgery for malignant disease to reduce the risk of VTE. However, to develop more secure evidence-based treatment guidelines, more research is required to show that further extension of anticoagulant treatment beyond hospitalization will decrease VTE risk to an even greater extent. 


\section{Prophylaxis in medical patients with cancer}

The ability to identify medical patients with cancer at greatest risk for thrombosis would facilitate more effective use of thromboprophylaxis. There are two main clinical settings that physicians should consider. The first involves the ambulatory patient who is receiving chemotherapy or radiotherapy, and the second is the cancer patient that is bedridden for an extended period of time. Interestingly, the Fundamental Research in Oncology and Thrombosis (FRONTLINE) survey showed that cancer patients under medical management do not commonly receive thromboprophylaxis (Wolff 2003). In such cancer patients, the cause of this risk and the timing of risk assessment are indistinct because risk factors are multiple and ever changing. For example, the incidence of thrombosis is increased with chemotherapy and hormonal therapy, and also with newer targeted anti-cancer agents such as antiangiogenic and cytokine therapy. For example, in breast cancer, both tamoxifen and cytotoxic chemotherapy appear to increase the risk for VTE independently. In a review of 2673 breast cancer patients in trials organized by the Eastern Cooperative Oncology Group, venous and arterial thromboses were significantly more common among women receiving chemotherapy plus hormonal therapy than in controls (Saphner et al 1991). In a double blind clinical trial, 311 patients with metastatic breast cancer were randomized to receive either very low dose warfarin (international normalized ratio [INR] of 1.3 to 1.9) or placebo while they were receiving chemotherapy (Levine et al 1994). This study showed an $85 \%$ relative reduction in risk of thrombosis in patients randomized to receive warfarin $(p=0.031)$ with no increase in risk of bleeding complications.

\section{Prophylaxis for central vein catheter thrombosis}

Central venous catheters (CVC) are used commonly for the administration of chemotherapeutic agents, blood components, or parenteral nutrition in cancer patients. The incidence of catheter-associated VTE reported in studies enrolling cancer patients is highly variable ranging from as low as $4 \%$ to as high as $66 \%$ (Balestreri et al 1995; Bona 1999; Karthaus et al 2006). Early studies revealed high rates of CVC-related thrombosis and significant reductions in such thrombosis with antithrombotic prophylaxis compared with no treatment (Bern et al 1990; Monreal et al 1996). In a recent large, multinational, double blind, placebo- controlled trial the efficacy and safety of dalteparin was evaluated for the prevention of clinically relevant CVCrelated thrombosis in cancer patients receiving chemotherapy via catheter (Karthaus et al 2006). This study showed that the risk of CVC-related thrombosis was 3.7\% in dalteparin-treated versus $3.4 \%$ in the placebo-treated patients. Not only was there no difference in risk of thrombosis, but there was also no difference in the time to onset of this complication between patients on dalteparin versus placebo. The low rates of thrombosis observed in recent studies may be a reflection of newer catheter design, improved catheter care or a shorter period of hospitalization, which has become common practice in the past decade. Anticoagulation is not recommended for routine prophylaxis of catheter related thromboembolic complications in cancer patients (Geerts et al 2004). However, this recommendation may change if a subgroup of cancer patients can be identified with a higher risk for development of CVC-related thrombosis.

\section{Management of cancer associated thrombosis}

The goal of therapy in cancer patients with VTE is to improve symptoms of VTE, reduce risk of recurrent VTE, prevent pulmonary embolism, and decrease the risk of postthrombotic syndrome. Management of VTE in cancer patients is problematic. Risk of recurrent thromboembolism and of anticoagulant treatment-related bleeding should be considered during treatment planning.

\section{Initial treatment of thrombosis in cancer patients}

The standard regimen for treatment of VTE in cancer patients involves the initial administration of LMWH or UFH for approximately 1 week followed by long-term oral anticoagulant therapy with a vitamin $\mathrm{K}$ antagonist, such as warfarin, to maintain an INR of between 2.0 and 3.0. Based on the results of several randomized controlled trials, LMWH has replaced UFH as the initial treatment in the majority of the patients with acute VTE. This is because large meta-analyses of many clinical trials conducted in inpatient and outpatient settings have revealed that LMWH administered subcutaneously is at least as safe and effective as UFH administered intravenously, and LMWH usually requires no laboratory monitoring and is associated with a reduced risk of developing heparin induced thrombocytopenia and thrombosis, and osteoporosis (Gould 
et al 1999; Dolovich et al 2000; van den Belt et al 2000). LMWH is more convenient to administer than UFH because it can be given by patients to themselves using once or twice daily subcutaneous injection with weight-adjusted dosing on an outpatient basis. For these reasons, LMWH has become the drug of choice for the initial therapy for VTE. Current guidelines from the American College of Chest Physicians (ACCP) suggest that LMWH may also be the treatment of choice for the long-term (for example for 3-6 months) treatment of VTE under certain circumstances such as when vitamin $\mathrm{K}$ antagonists are contraindicated or impractical, in patients with thrombosis in spite of therapeutic levels of vitamin $\mathrm{K}$ antagonists and in patients at especially high risk of recurrence. An example of the latter would be patients with malignancy-associated VTE in whom LMWH regimens may be more effective and safer (Buller et al 2004).

\section{Long-term management of thrombosis in cancer patients}

Recurrent VTE is common following the first such episode in cancer patients. Clinical studies have shown that cancer patients diagnosed with VTE have an increased risk of developing recurrent thromboembolic and bleeding complications with antithrombotic treatments, compared with patients having VTE without cancer (Prandoni et al 2002). Problems associated with the long-term treatment of VTE in the setting of cancer using oral anticoagulants are related to the fact that cancer patients commonly manifest variability in dietary intake, malnutrition, liver dysfunction related to the presence of metastases and other factors, and nausea and vomiting due to chemotherapy toxicity. Cancer patients are also more likely to ingest a variety of drugs capable of interacting with and altering the dose requirement of oral anticoagulants. Such uncertainties might be overcome with long-term use of LMWH. In a multicenter, randomized clinical trial, subcutaneous therapeutic LMWH (tinzaparin) administered for 84 days versus initial treatment using UFH for 5 days together with long-term warfarin sodium given to 84 days for the treatment of high risk patients (including cancer patients) with objectively diagnosed proximal deep-vein thrombosis were compared. In this study, long-term tinzaparin administration was effective compared with UFH/warfarin sodium and was clinically and statistically significantly safer than UFH/longterm oral anticoagulants due to a lower frequency of hemorrhagic complications (Hull et al 2003). The results of two randomized trials have been published comparing LMWH versus warfarin for the prevention of recurrent VTE in cancer patients (Meyer et al 2002; Lee et al 2003). In a multicenter randomized trial, 3 months of treatment with enoxaparin was compared with warfarin and a combined outcome of bleeding plus recurrent VTE was evaluated (Meyer et al 2002). Of the 71 evaluable patients receiving warfarin, 15 (21\%) experienced a major hemorrhage or recurrent VTE compared with 7 (10.5\%) of the 67 patients assigned to receive enoxaparin; this difference approached statistical significance $(p=0.09)$. The Randomized Comparison of Low-Molecular-Weight Heparin versus Oral Anticoagulant Therapy for the Prevention of Recurrent Venous Thromboembolism in Patients with Cancer (CLOT) trial was a multicenter, randomized, open label clinical trial that investigated whether dalteparin was more effective and safer than oral anticoagulant therapy in preventing recurrent thromboembolism in cancer patients who had an episode of acute VTE (Lee et al 2003). During the six-month study period, 27 of the 336 patients in the dalteparin group had recurrent VTE compared with 53 of the 336 patients in the oral-anticoagulant group ( $\mathrm{p}=0.002)$. Thus, the probability of recurrent VTE at six months was $17 \%$ in the oralanticoagulant group and $9 \%$ in the dalteparin group. No significant difference between the dalteparin group and the oral anticoagulant group was found in the rate of total bleeding episodes or of major bleeding. The CLOT study showed that dalteparin demonstrated superior safety and efficacy compared with an oral vitamin $\mathrm{K}$ antagonist in for the prevention of recurrent VTE in cancer patients.

\section{Anticancer effect of anticoagulants}

The first report of a possible beneficial effect of an anticoagulant on the natural history of malignancy was published in 1964 for the vitamin K antagonist, warfarin (Michaels 1964). The first prospective randomized trial designed to test the ability of warfarin to influence cancer survival was published in 1981 (Zacharski et al 1981). This study showed that survival of patients with small-cell lung cancer (SCLC) was prolonged significantly upon the addition of warfarin to standard combination chemotherapy plus radiation therapy. The median survival for control patients was 24 weeks and for warfarin-treated patients 50 weeks. Warfarin-treated patients also demonstrated a significantly increased time to first evidence of disease progression. Recently, several randomized trials have examined the effect of LMWH on the survival of cancer 
patients with or without VTE (Altinbas et al 2004; Kakkar et al 2004; Klerk et al 2005; Lee et al 2005). The results of all these clinical studies showed that LMWH provided a survival benefit, particularly in patients in the early stages of malignant disease. The study of Altinbas and colleagues (2004) was well controlled and included only patients with SCLC. However, the remaining three studies were not controlled for tumor type, disease stage, performance status or standard anti-tumor treatment. Thus, it remains to be determined whether patients with tumor types other than SCLC will respond to this treatment approach. The fact that the coagulation mechanism is intimately involved in wound healing and tissue remodeling and organogenesis provides a conceptual basis for postulating that it is involved in the disorderly growth characteristic of malignancy (Zacharski 2003). Considering the substantial number of studies in experimental tumor systems that support the concept that the coagulation mechanism promotes malignant growth and the fact that numerous drugs that are relatively non-toxic are currently available for testing, it is perhaps surprising that more attention has not been paid to exploring this novel cancer treatment paradigm. Further studies designed to clarify possible anti-tumor effects of anti-thrombotic drugs, and especially the LMWHs, and to explore the heterogeneous pathophysiological mechanisms that pertain to different tumor types are awaited.

\section{Summary and future directions}

LMWH is the treatment of choice for the initial treatment of patients with VTE occurring in the setting of active malignancy. The optimal duration of LMWH therapy for prevention of recurrent VTE is controversial and should be tailored to the needs of the individual patient. The advent of new anti-thrombotic agents may provide more treatment options in the future. However, clinical trials must be designed carefully with full consideration given not only to effects of treatment on VTE risk and toxicity of therapy, but also to the possibility that agents with varying mechanisms of action may alter the natural history of malignancies in which that particular mechanism may drive tumor progression. The ideal anticoagulant should have wide therapeutic window, low risk of bleeding, no serious side effects, and no or few drug interactions while being effective in reducing VTE, relatively easy to administer and follow (no laboratory monitoring), and rapidly reversible. Progress in optimizing VTE prevention and treatment in the setting of cancer is anticipated.

\section{Disclosure}

Dr Er reports no issues of conflict of interest. Dr Zacharski has been a consultant to AstraZeneca, Bayer, Bristol-Meyers Squibb, Pharmion and Sanofi-Aventis pharmaceutical companies. There are no other connections of any kind to industry or private interests.

\section{References}

Alcalay A, Wun T, Khatri V, et al. 2006. Venous thromboembolism in patients with colorectal cancer: incidence and effect on survival. $J$ Clin Oncol, 24:1112-18.

Altinbas M, Coskun HS, Er O, et al. 2004. A randomized clinical trial of combination chemotherapy with and without low-molecular-weight heparin in small cell lung cancer. $J$ Thromb Haemost, 2:1266-71.

Balestreri L, De Cicco M, Matovic M, et al. 1995. Central venous catheterrelated thrombosis in clinically asymptomatic oncologic patients: a phlebographic study. Eur J Radiol, 20:108-11.

Bergqvist D, Burmark U, Flordal P, et al. 1995. Low molecular weight heparin started before surgery as prophylaxis against deep vein thrombosis:2500 versus $5000 \mathrm{XaI}$ units in 2070 patients. Br J Surg, 82:496-501.

Bergqvist D, Agnelli G, Cohen A, et al. 2002. Duration of prophylaxis against venous thromboembolism with enoxaparin after surgery for cancer. $N$ Engl J Med, 346:975-80.

Bern M, Lokich J, Wallach S, et al. 1990. Very low doses of warfarin can prevent thrombosis in central venous catheters. A randomized prospective trial. Ann Intern Med, 112:423-8.

Bona R. 1999. Thrombotic complications of central venous catheters in cancer patients. Semin Thromb Hemost, 25:147-55.

Buller HR, Agnelli G, Hull RD, et al. 2004. Antitrombotic therapy for venous thromboembolic disease. Chest, 126:421S-428S

Chew H, Wun T, Harvey D, et al. 2006. The incidence of venous thromboembolism and its effect on survival among patients with common cancers. Arch Intern Med, 166:458-64.

Clagett GP, Reisch JS. 1988. Prevention of venous thromboembolism in general surgical patients. Ann Surg, 208:227-40.

Dolovich LR, Ginsberg JS, Douketis JD, et al. 2000. A meta-analysis comparing low-molecular-weight heparins with unfractionated heparin in the treatment of venous thromboembolism:examining some unanswered questions regarding location of treatment, product type, and dosing frequency. Arch Intern Med, 160:181-8.

Galster H, Kolb G, Kohsytorz A, et al. 2000. The pre-, peri-, and postsurgical activation of coagulation and the thromboembolic risk for different risk groups. Thromb Res, 100:381-8.

Geerts WH, Pineo GF, Heit JA, et al. 2004. Prevention of venous thromboembolism: the Seventh ACCP Conference on Antithrombotic and Thrombolytic Therapy. Chest, 126:338S-400S.

Gould MK, Dembitzer AD, Doyle RL, et al. 1999. Low-molecular-weight heparins compared with unfractionated heparin for treatment of acute deep venous thrombosis. A meta-analysis of randomized, controlled trials. Ann Intern Med, 130:800-9.

Heit JA, Silverstein MD, Mohr DN, et al. 1999. Predictors of survival after deep vein thrombosis and pulmonary embolism: A population based, cohort study. Arch Intern Med, 159:445-53.

Hettiarachchi RJ, Lok J, Prins MH, et al. 1998. Undiagnosed malignancy in patients with deep vein thrombosis: incidence, risk factors, and diagnosis. Cancer, 83:180-5.

Hull RD, Pineo GF, Mah AF, et al; for the LITE Investigators. 2003. A randomized trial evaluating long-term low-molecular-weight heparin therapy for three months versus intravenous heparin followed by warfarin sodium. J Thromb Haemost, 1(Suppl 1):OC395. 
Kakkar AK, Levine MN, Kadziola Z, et al. 2004. Low molecular weight heparin, therapy with dalteparin, and survival in advanced cancer: The Fragmin Advanced Malignancy Outcome Study (FAMOUS). $J$ Clin Oncol, 22:1944-8.

Karthaus M, Kretzschmar A, Kroning H, et al. 2006. Dalteparin for prevention of catheter-related complications in cancer patients with central venous catheters: final results of a double-blind, placebocontrolled phase III trial. Ann Oncol, 17:289-96.

Khorana AA. 2003. Malignancy, thrombosis and Trousseau: the case for an eponym. J Throm Haemost, 1:2463-5.

Klerk CPW, Smorenburg SM, Otten JMMB, et al. 2005. The effect of low molecular weight heparin on survival in patients with advanced malignancy. J Clin Oncol, 23:2130-5.

Lee AYY, Levine MN. 2002. Cancer and thrombosis: Current concepts in patient care. Connecticut: Chase Medical Communications.

Lee AY, Levine MN, Baker RI, et al. 2003. Low molecular weight heparin versus a coumarin for the prevention of recurrent venous thromboembolism in patients with cancer. $N$ Engl J Med, 349:146-53.

Lee A, Rickles FR, Julian JA, et al. 2005. Randomized comparison of low molecular weight heparin and coumarin derivatives on the survival of patients with cancer and venous thromboembolism. J Clin Oncol, 23:2123-9.

Levine M, Hirsh J, Gent M, et al. 1994. Double-blind randomised trial of a very-low-dose warfarin for prevention of thromboembolism in stage IV breast cancer. Lancet, 343:886-9.

Levitan N, Dowlati A, Remick SC, et al. 1999. Rates of initial and recurrent thromboembolic disease among patients with malignancy versus those without malignancy. Risk analysis using Medicare claims data. Med (Baltimore), 78:285-91.

Lip GY, Chin BS, Blann AD. 2002. Cancer and the prothrombotic state. Lancet Oncol, 3:27-34.

Meyer G, Marjanovic Z, Valcke J, et al. 2002. Comparison of lowmolecular-weight heparin and warfarin for the secondary prevention of venous thromboembolism in patients with cancer: a randomized controlled study. Arch Intern Med, 162:1729-35.

Michaels L. 1964. Cancer incidence and mortality in patients having anticoagulant therapy. Lancet, 22:832-5.
Mismetti P, Laporte S, Darmon JY, et al. 2001. Meta-analysis of low molecular weight heparin in the prevention of venous thromboembolism in general surgery. Br J Surg, 88:913-30.

Monreal M, Alastrue A, Rull M, et al. 1996. Upper extremity deep venous thrombosis in cancer patients with venous access devices - prophylaxis with a low molecular weight heparin (Fragmin). Thromb Haemost, 75:251-3.

Monreal M, Lensing AWA, Prins MH, et al. 2004. Screening for occult cancer in patients with acute deep vein thrombosis and pulmonary embolism. J Throm Haemost, 2:876-81.

Prandoni P, Lensing A, Piccioli A, et al. 2002. Recurrent venous thromboembolism and bleeding complications during anticoagulant treatment in patients with cancer and venous thrombosis. Blood, 100:3484-8

Rasmussen MS, Wille-Jorgensen P, Jorgensen LN, et al. 2003. Prolonged thromboprophylaxis with low molecular weight heparin (dalteparin) following major abdominal surgery for malignancy [abstract]. Blood, 102:186.

Saphner T, Tormey DC, Gray R. 1991. Venous and arterial thrombosis in patients who receive adjuvant therapy for breast cancer. J Clin Oncol, 9:286-94.

Sorensen HT, Mellemkjaer L, Olsen JH, et al: 2000. Prognosis of cancers associated with venous thromboembolism. $N$ Engl J Med, 343:184650.

van den Belt AG, Prins MH, Lensing AW, et al. 2000. Fixed dose subcutaneous low molecular weight heparins versus adjusted dose unfractionated heparin for venous thromboembolism. Cochrane Database Syst Rev, CD001100.

Wolff R. 2003. Are patients with cancer receiving adequate thromboprophylaxis? Results from FRONTLINE. Cancer Treat Rev, 29(Suppl 2):7-9.

Zacharski LR, Henderson WG, Rickles FR, et al. 1981. Effect of warfarin on survival in small cell carcinoma of the lung. JAMA, 245:831-5

Zacharski LR. 2003. Malignancy as a solid-phase coagulopathy: implications for the etiology, pathogenesis, and treatment of cancer. Semin Thromb Hemost, 29:239-46. 